\title{
Stability analysis of a model of atherogenesis: An energy estimate approach
}

\author{
A.I. Ibragimov ${ }^{\mathrm{a}}$, C.J. McNeal ${ }^{\mathrm{b}}$, L.R. Ritter ${ }^{\mathrm{c} *}$ and J.R. Walton ${ }^{\mathrm{d}}$ \\ ${ }^{a}$ Department of Mathematics, Texas Tech University, Lubbock, TX 79409, USA; ${ }^{b}$ Division of \\ Cardiology and Department of Pediatrics, Division of Endocrinology, Department of Internal \\ Medicine, Scott and White, Temple, TX 76508, USA; ${ }^{c}$ Department of Mathematics, Southern \\ Polytechnic State University, Marietta, GA 30060, USA; ${ }^{d}$ Department of Mathematics, \\ Texas A \& M University, College Station, TX 77843-3368, USA
}

(Received 29 August 2007; final version received 12 December 2007)

\begin{abstract}
Atherosclerosis is a disease of the vasculature that is characterized by chronic inflammation and the accumulation of lipids and apoptotic cells in the walls of large arteries. This disease results in plaque growth in an infected artery typically leading to occlusion of the artery. Atherosclerosis is the leading cause of human mortality in the US, much of Europe, and parts of Asia. In a previous work, we introduced a mathematical model of the biochemical aspects of the disease, in particular the inflammatory response of macrophages in the presence of chemoattractants and modified low density lipoproteins. Herein, we consider the onset of a lesion as resulting from an instability in an equilibrium configuration of cells and chemical species. We derive an appropriate norm by taking an energy estimate approach and present stability criteria. A bio-physical analysis of the mathematical results is presented.
\end{abstract}

Keywords: atherosclerosis; atherogenesis; chemotaxis; stability analysis; energy estimate

2000 Mathematics Subject Classification: 35K55; 92C17; 92C50

\section{Introduction}

Atherosclerosis is a disease of the vasculature that is characterized by an accumulation of lipid-laden immune cells and apoptotic cells in the arterial wall. Recently, the authors proposed a mathematical model of the early stages of the disease [11]. The model is based on a view of the process consistent with the paradigm of Russell Ross that atherosclerosis is an inflammatory disease [18]. Throughout the West and in parts of Asia, coronary artery disease (which is caused primarily by atherosclerosis) is the leading cause of human mortality. An enhanced understanding of the disease, its progression, risk factors, precursors and indicators, is essential to the development of more effective treatment and prevention strategies. Mathematical modelling is an important instrument in this endeavour.

The following section contains a description of the disease forming the basis for the mathematical model presented in Ref. [11]. That general model is briefly reiterated here for convenience. One view of the onset of a lesion is that it results from an instability

*Corresponding author. Email: 1ritter@spsu.edu 
in a healthy uniform state. The notion of aggregation resulting from an unstable perturbation is classical in the study of chemotactic organisms ( $c f$. Keller and Segel 1971; [13]). In Ref. [12], the current authors offer a linear stability analysis of a simplified system of equations and obtain a physically relevant criterion for instability via a standard perturbation analysis. In the current work, we consider a somewhat more complex system of equations. Through an analysis of energy estimates, we obtain a sufficient condition underwhich the uniform healthy state is stable. This is presented in full in Sections 4 and 5 and a discussion follows.

\section{The disease process}

Recent decades have seen an increase in the use of mathematics as a tool for understanding biological and medical phenomena. These include numerous studies of the cardiovascular system with attention to mechanical properties of biological soft tissue, the fluid dynamics of blood and biochemical properties of the vasculature. The current work is concerned primarily with the last of these: biochemical aspects of the arterial wall (both healthy and diseased). However, a comprehensive view of atherosclerosis will ultimately require integration of these various modelling perspectives. The inflammation underlying atherosclerosis involves the response of certain cells (immune cells) to the chemical environment (the biochemical perspective). The formation of an atherosclerotic lesion causes the tissue to stiffen and change its shape (the mechanical properties change). The flow of blood is affected by the altered geometry of the artery (fluid dynamical issues). The properties of the blood flow directly affect the dispersal of chemical and cellular species near the walls of the artery and (perhaps more importantly) transport of species across the arterial wall.

The earliest stages of disease in the arterial wall requires a change in permeability of the interface between the blood flow and the tissue that comprises that actual wall of the artery (so called endothelial dysfunction). It has long been believed that the dynamics of the fluid flow is an important factor in these stages. In the 1950s and 60s it was thought that perhaps shear stress on the wall of the artery had an erosive effect. However, atherosclerotic lesions are frequently observed in parts of the tissue subjected to low or oscillating shear stress, for example the opposing wall of an arterial bifurcation. In 1969 and 1971, Caro, Fitz-Gerald and Schroter correlated the onset of arterial disease with a shear dependent mass transfer mechanism and showed that this is consistent with the observation of higher incidence of lesions at the sites of reduced shear stress [2]. The exchange of cellular and chemical species across the lining of the arterial wall is key to the onset and progression of atherosclerosis.

Russell Ross divides the lesions of atherosclerosis into three categories. The first is called a fatty streak, and is characterized by a collection of immune cells that are lipidladen. This type of lesions may be found through out the arterial tree (commonly at the site of changes in the blood flow), and are seen in humans even in early childhood [17]. The more advanced lesions, the fibrofatty lesion and the fibrous plaque, are observed in the large, thick-walled arteries such as the coronary, femoral, or cerebral arteries and the abdominal aorta. Such arteries can be described as thick tubes consisting of three distinct layers. The outermost layer is the adventitia which contains thick bundles of collagen fibers aligned primarily in the axial direction. The adventitia provides axial strength and prevents overstretch or rupture. The middle layer of the artery is the thickest and consists of layered smooth muscle cells (SMCs) in an extracellular matrix of collagen and elastin. This layer is called the media. In it, the SMCs - in circumferential layers - provide the artery with resistance to loads both radially and axially [9]. The muscular nature of the 
large arteries allows the arteries to contract, aiding the heart in pushing blood to the extremities. The innermost layer is the intima. This is the smallest layer of the artery, making up less that $10 \%$ of the thickness, but it is the layer most affected by the disease of atherosclerosis. A thin membrane called the internal elastic lamina (IEL) separates the intima from the media. At the lumen, there is a monolayer of endothelial cells lining the intima and providing the crucial interface between the arterial wall and the blood stream. Between the endothelial layer and the IEL is a subendothelial intima (SI) containing proteoglycans and thin collagen fibrils. The intima is the site of atherosclerotic lesions. The fibrofatty lesions contain lipid-laden immune cells just as in the fatty streak. These more advanced lesions, however, also consist of layers of SMCs in a poorly developed connective tissue matrix of collagen, elastin and proteoglycans. Finally, the fibrous plaque is characterized by a dense cap of fibrous tissue covering the fatty inner lesion (the advanced inner lesion may also contain nectrotic tissue). This cap may be comprised primarily of SMCs and connective tissue, or it may contain layers of lipid-laden immune cells between layers of SMCs. A uniform and dense cap can offer a stabilizing effect to the lesion. By contrast, a nonuniform or thin cap may result in sudden rupture of the lesion. The medical consequences of plaque rupture are often catastrophic leading to heart attack, stroke, or sudden death.

Most advanced atherosclerotic lesions consist of a lipid core surrounded by a fibrous cap of SMCs and connective tissue. This cap formation is considered by the authors in Ref. [11] and is included in the general model. In the current work, we focus on the earliest stages of lesion formation. During the process of endothelial dysfunction, the transport of low density lipoproteins (LDL), immune cells and potentially bacteria or viral particles into the SI occurs. Endothelial dysfunction may also cause endothelial cells to secrete procoagulant chemicals and chemical signals summoning immune response cells. Following this initial dysfunction, disease can result from chemical modification of LDL particles, disruption of the normal immune function, continued inflammation and lesion growth $[3,18]$.

Lipoproteins are micellar particles produced by the liver and intestines which contain regulatory proteins that direct the blood trafficking of cholesterol and other lipids to various cells in the body. There are four major classes of lipoprotein: very low-density lipoprotein, intermediate-density lipoprotein, low-density lipoprotein (the 'bad cholesterol' containing particle), and high-density lipoprotein (HDL, the 'good cholesterol' containing particle). The bulk of cholesterol is contained within the latter two particles. The lipoprotein structure consists of a lipid core containing cholesterol esters and triglicerides, and a coat that is composed of regulatory surface proteins, unesterified cholesterol, phospholipids and a variety of other minor components that may include molecules and proteins associated with antioxidant defences. LDL particles transport cholesterol that is needed for various cellular functions such as cell membrane formation and hormone synthesis. About $60-70 \%$ of the total body cholesterol is contained in the LDL particles. HDL particles account for most of the remaining cholesterol. The function of the HDL particles appears to be involved with the return of excess lipids from tissues to the liver for subsequent processing (a process referred to as reverse transport). Many studies have unequivocally shown that elevated blood levels of LDL cholesterol confer a higher risk of developing cardiovascular disease. Although LDL particles are not found in atherosclerotic plaques, oxidatively modified LDL particles are.

This process of LDL modification is obligatory in the formation of the atherosclerotic plaque [7,20]. In 1977, Goldstein et al. [7] discovered that certain immune cells, in particular macrophages, have a high affinity for oxidatively-modified LDL but not native 
LDL. This results in trapping of cholesterol within the arterial wall. Macrophages engorged with lipids are referred to as foam cells. Unable to perform their normal duty of degrading debris, these lipid-laden cells accumulate and signal other immune cells to the site in a cascading progression to plaque growth.

Various immune cells are responsible for the degradation of apoptotic cells and for combating threatening agents such as certain bacteria or viruses throughout the human body. Monocytes, a species of white blood cells, are one such immune cell that are found in the circulating blood. When immune response is required, it is typically mediated by the excretion of various chemical signals. One of the many functions of endothelial cells is the signalling of immune response cells during time of injury. Changes in the permeability of the endothelial layer and subsequent deposition of lipids in the intima cause an up-regulation of chemoattractants secreted by these cells including monocyte chemotactic protein 1 , interleukin- 8 and macrophage colony stimulating factor [17]. Once in the artery wall, monocytes differentiate into macrophages which are phagocytic cells that seek out and engulf apoptotic or foreign bodies. It is now understood that macrophages become corrupted in the presence of oxidized LDL and are a major player in the inflammatory process of atherosclerosis [19]. LDL particles in the native state do not attract macrophages. However, once LDL is oxidized it is recognized by the scavenger receptor on the surface of the macrophages $[7,16]$. Attracted by oxidized LDL, the macrophages in the artery wall attempt to internalize the lipoprotein particles. This results in an accumulation of cholesterol esters and subsequent transformation of a macrophage into a foam cell. In this lipid-laden state, the macrophage is incapable of functioning normally. Dead or apoptotic cells and other debris (including foam cells) are allowed to build up. In response, chemical signals are secreted by the foam cells and endothelial cells to summon more immune cells to the site. Additional macrophages then enter into the region. The chemical mediators of inflammation can increase binding of oxLDL to cells in the arterial wall [8]. Hence, the new macrophages become engorged with oxLDL and the cycle of chemical signalling continues.

In addition to foam cells, apoptotic macrophages are regularly found in atherosclerotic plaques [14]. Apoptosis of cells within a plaque, macrophage and others, is found to have both stabilizing as well as destabilizing effects [5,21]. Phagocytosis of apoptotic cells (not necessarily macrophages) may induce resistance to foam cells formation among macrophages. This occurs when during phagocytosis, the macrophage takes in high levels of membrane-derived cholesterol as opposed to lipoprotein-derived cholesterol. In Cui et al., the authors report that ingestion of apoptotic cells induced a survival response in the macrophages in their experiments [5]. It has also been reported that apoptotic macrophages can lead to destabilization, especially in the later stages of plaque formation. In vulnerable plaques, those that are advanced and have the potential for rupture, it has been found that macrophage apoptosis induced by accumulation of large amount of LDL cholesterol can lead to plaque rupture and acute occlusion [21]. Because apoptosis is an influential factor in atherosclerosis, the model proposed herein is constructed so as to account for the impact of both healthy and apoptotic macrophages. For a simple schematic of the interactions considered in this work, see Figure 1.

\section{The general mathematical model}

The model proposed in Ref. [11] consists of a number of (generalized) cellular and chemical species inherent in the disease and a mathematical description of their interactions and evolution. The cellular species fall into three categories: 


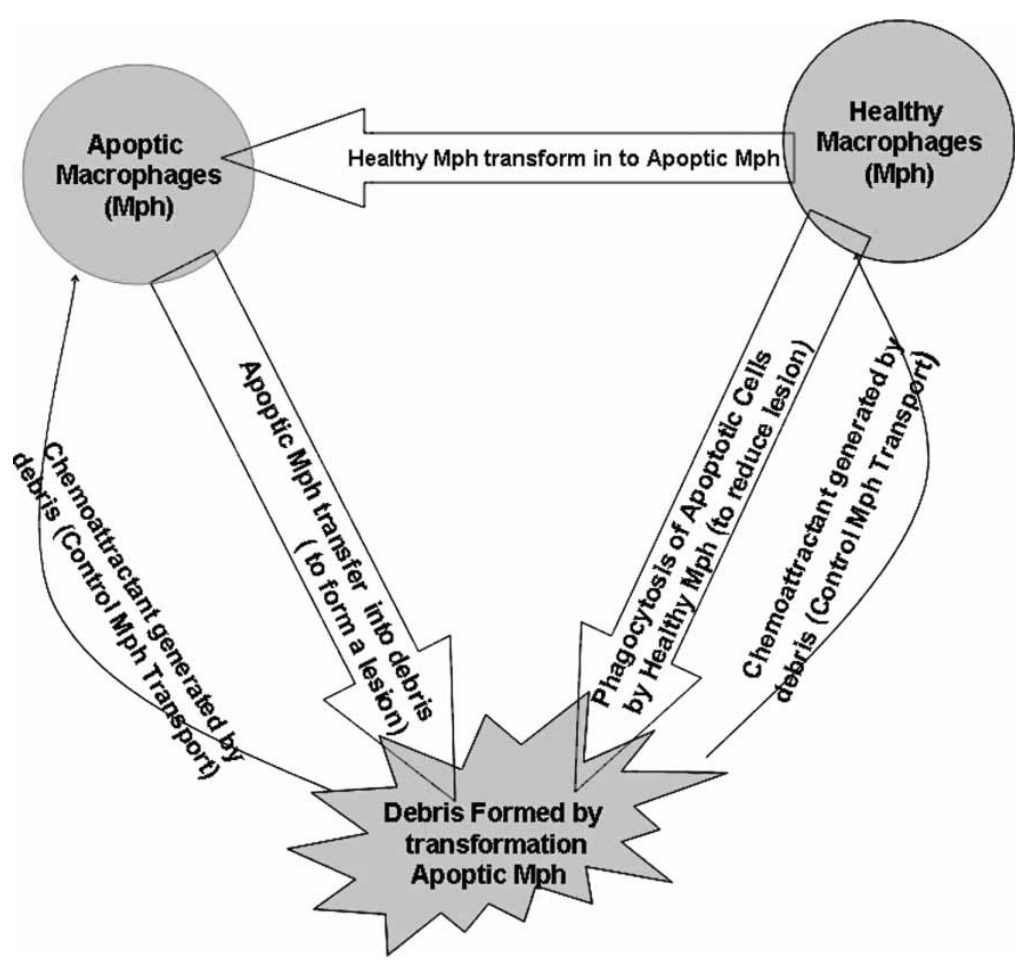

Figure 1. Schematic of immune cell interaction and possible lesion formation.

- Immune cells: cells involved in the immune response. These are primarily monocyte derived macrophages but may also include T-lymphocytes.

- SMCs: this generalized species can also include cells such as fobroblasts which are responsible for production of the extra cellular matrix.

- Debris: this is a broad category that may include cells that are dead or apoptotic, necrotic tissue, and foam cells.

Similarly, the chemical species are one of three main types:

- Chemoattractant: this is intended to denote any chemical that induces positive chemotaxis (of immune cells or SMCs). Here, no distinction is made among various types of chemoattractants such as macrophage colony stimulating factor, monocyte chemotactic protein, interleukin-1 and others.

- Native lipoproteins: LDL cholesterol (in a nonoxidized state) is the primary species of interest. However, it can also be expanded to include HDL, the role of which is included in the model of LDL oxidation provided by Cobbold Sherratt and Maxwell[4].

- Oxidized LDL.

For each species, an evolution equation is derived through the classical approach of imposing a mass balance in an arbitrary control volume and subsequent reduction to a pointwise statement. We do not consider here the volume of a lesion but rather the concentration of each species at any point. The primary means of transport for the debris 
species as well as for all three chemical species is assumed to be simple diffusion. However, for the immune cells and SMCs, the highly interactive nature of their transport in the inflammatory process is accounted for primarily through chemotaxis. Hence, the model given by Keller and Segel in 1971 [13] is employed to describe this process.

If we let $n_{1}, n_{2}$ and $n_{3}$ denote the concentration of immune cells, SMCs and debris respectively and $c_{1}, c_{2}$ and $c_{3}$ the concentration of chemoattractant, native lipoproteins and oxidized LDL respectively. Define the flux, $\mathbf{J}_{n_{i}}$ of species $n_{i}$ that exhibits chemotactic movement in response to chemical species $c_{j}$, diffusive transport and transport sensitivity to gradients of the other cellular species and debris by

$$
\mathbf{J}_{n_{i}}=-\mu_{i} \nabla n_{i}+\sum_{j \neq i} \xi_{i j}\left(n_{j}, n_{i}\right) \nabla n_{j}+\sum_{j=1} \chi_{i j}\left(c_{j}, n_{i}\right) \nabla c_{j} .
$$

The coefficients $\xi_{i j}\left(n_{j}, n_{i}\right)$ and $\chi_{i j}\left(c_{j}, n_{i}\right)$ are called the tactic sensitivity functions. These coefficients characterize the impact of dragging forces imposed by the gradient of the chemo-attractor. They are typically assumed to be linear in the cellular species $n_{i}$, $[10,13,15]$ which leads to an advective term in the resulting equation. The coefficients $\mu_{i}$ characterize the general mobility of cells due to random motion. There results a system of partial differential equations for the species $n_{j}(x, t)$ of the form

$$
\frac{\partial n_{i}}{\partial t}=-\nabla \mathbf{J}_{n_{i}}+r_{n_{i}}
$$

with $r_{n_{i}}$ denoting a net production term for species $n_{i}$. Each of the chemical species $c_{i}$ is subject to random diffusion and some net production. Taking the diffusion to be Fickian, the flux field for $c_{i}$ has the form $-\nu_{i} \nabla c_{i}$ with diffusion coefficient $\nu_{i}$. In general, $\nu_{i}$ may be spatial dependent. Herein, we will take $\nu_{i}$ as well as $\mu_{i}$ to be constants. Letting $q_{c_{i}}$ denote the net production term for species $c_{i}$ we arrive at the equation governing the $i$ th chemical species

$$
\frac{\partial c_{i}}{\partial t}=\nabla \cdot\left(\nu_{i} \nabla c_{i}\right)+q_{c_{i}}
$$

These governing equations are assumed to hold on some domain $\Omega$. The model is completed by specification of the domain, each of the production and tactic sensitivity terms and boundary and initial conditions.

As stated, this paper is devoted to a stability analysis to aquire criteria that will ensure that an equilibrium configuration - here viewed as corresponding to a healthy state - is stable. In the following section, this is carried out.

\section{Linearization of the original nonlinear system of reaction diffusion equations}

We consider a subsystem of (2)-(3), consisting of four species - two subspecies of immune cells, debris and a general chemoattractant. This is in a sense an extension of the stability analysis presented in Ref. [12]. Here, however, we consider both a normal healthy concentration of immune cells and a concentration of immune cells that are becoming corrupted but are not yet dead or foam cells. By allowing for two phases of immune cells, we are able to better account for the complex process of inflammation - the detrimental 
affects that runaway inflammation can result in, as well as the benefit of healthy, moderated inflammation. We introduce the convenient notation:

- $m$ concentration of healthy macrophages

- $\hat{m}$ concentration of apoptotic or otherwise unhealthy macrophages

- $d$ concentration of debris including dead cells, foam cells and the contents of a plaque and

- $c$ the concentration of chemoattractant.

The governing equations considered here are specified by

$$
\begin{aligned}
\partial_{t} m & =\mu_{\mathrm{u}} \nabla^{2} m-\chi_{1}^{0} \nabla \cdot\left(\frac{m}{c} \nabla c\right)-g_{0}(m, \hat{m})+M_{0} \\
\partial_{t} \hat{m} & =\mu_{\mathrm{v}} \nabla^{2} \hat{m}-\chi_{2}^{0} \nabla \cdot\left(\frac{\hat{m}}{c} \nabla c\right)+g_{0}(m, \hat{m})-F(\hat{m}) \\
\partial_{t} d & =\mu_{\mathrm{w}} \nabla^{2} d-g_{1}(m, d)+F(\hat{m}) \\
\partial_{t} c & =\mu_{\mathrm{z}} \nabla^{2} c-(a m+\hat{a} \hat{m}) c+f(d) d .
\end{aligned}
$$

These hold on some domain $\Omega$ with homogenous Neumann conditions on the boundary $\partial \Omega$. Because we consider this boundary condition, we introduce the source term for healthy macrophages $M_{0}$. In general, this source function would depend on the total concentration of macrophages as well as the levels of chemoattractant and LDL present. For simplicity, we assume that it is essentially constant over the ranges of these variables under consideration. Monocytes enter into the tissue from the blood stream. Once they are in the intima, they can differentiate to become macrophages. This source term captures this effect. We assume that all newly formed macrophages are of the healthy subtype. The function $g_{0}$ accounts for conversion of healthy macrophages into unhealthy macrophages, $F$ accounts for foam cell formation and $g_{1}$ is the immune response. If the range of changes in the variables $m, \hat{m}$ and $d$ are such that $g_{1}>F$ we consider the overall state of the system to be healthy. In general, the function $F$ depends on the level of modified LDL present. Since we are assuming here that the concentration of all LDL species is constant, we suppress this dependence at present.

We begin the analysis by letting $\left(m_{\mathrm{e}}, \hat{m}_{\mathrm{e}}, d_{\mathrm{e}}, c_{\mathrm{e}}\right)$ be the equilibrium solution of system (4) -(7). This leads immediately to the conditions

$$
\begin{aligned}
g_{0}\left(m_{\mathrm{e}}, \hat{m}_{\mathrm{e}}\right) & =M_{0} \\
F\left(\hat{m}_{\mathrm{e}}\right) & =g_{1}\left(m_{\mathrm{e}}, d_{\mathrm{e}}\right)=M_{0} \\
f\left(d_{\mathrm{e}}\right) d_{\mathrm{e}} & =\left(a m_{\mathrm{e}}+\hat{a} \hat{m}_{\mathrm{e}}\right) c_{\mathrm{e}}
\end{aligned}
$$

Next, we consider a perturbation of this equilibrium state. The perturbation variables $u, v$, $w$ and $z$ are introduced and are defined by

$$
m=m_{\mathrm{e}}+u, \quad \hat{m}=\hat{m}_{\mathrm{e}}+v, \quad d=d_{\mathrm{e}}+w, \quad \text { and } \quad c=c_{\mathrm{e}}+z .
$$


Substituting these into Equations (4)-(7) and keeping only linear terms results in the system that determines the new variables:

$$
\begin{gathered}
\partial_{t} u=\mu_{u} \nabla^{2} u-\chi_{u} \nabla^{2} z-A u-B v \\
\partial_{t} v=\mu_{v} \nabla^{2} v-\chi_{v} \nabla^{2} z+A u+(B-C) v \\
\partial_{t} w=\mu_{w} \nabla^{2} w-E u+C v-G w \\
\partial_{t} z=\mu_{z} \nabla^{2} z-H u-I v+K w-J z \\
\text { with } \quad \frac{\partial u}{\partial \vec{n}}=\frac{\partial v}{\partial \vec{n}}=\frac{\partial w}{\partial \vec{n}}=\frac{\partial z}{\partial \vec{n}}=0 \quad \text { on the boundary and } \\
u(x, 0)=u_{0}, \quad v(x, 0)=v_{0}, \quad w(x, 0)=w_{0}, \quad z(x, 0)=z_{0}
\end{gathered}
$$

The parameters appearing in this system are defined by:

$$
\begin{gathered}
\chi_{u}=\chi_{1}^{0} \frac{m_{\mathrm{e}}}{c_{\mathrm{e}}}, \quad \chi_{v}=\chi_{2}^{0} \frac{\hat{m}_{\mathrm{e}}}{c_{\mathrm{e}}}, \quad A=\partial_{m} g_{0}, \quad B=\partial_{\hat{m}} g_{0}, \quad C=F^{\prime}\left(\hat{m}_{\mathrm{e}}\right) \\
E=\partial_{m} g_{1}, \quad G=\partial_{d} g_{1}, \quad H=a c_{\mathrm{e}}, \quad I=\hat{a} c_{\mathrm{e}} \\
K=f\left(d_{\mathrm{e}}\right)+f^{\prime}\left(d_{\mathrm{e}}\right) d_{\mathrm{e}}, \quad \text { and } \quad J=a m_{\mathrm{e}}+\hat{a} \hat{m}_{\mathrm{e}},
\end{gathered}
$$

where $\partial_{\alpha}$ denotes the partial derivative with respect to the variable $\alpha$ and all functions are evaluated at the equilibrium values of $m-c$.

In the section to follow, we begin to study stability of the equilibrium solution $\left(m_{\mathrm{e}}, \hat{m}_{\mathrm{e}}, d_{\mathrm{e}}, c_{\mathrm{e}}\right)$. For ease of notation, let $\vec{U}(x, t)=(u, v, w, z)$. We define stability:

Definition. The equilibrium state is called asymptotically stable if every solution of the linearized Initial Boundary Value Problem (IBVP) (8)-(13) for the perturbation variables vanishes at infinity in the sense that there exists a positive functional

$$
\mathcal{F}(\vec{U})=\Phi(t) \quad \text { such that } \quad \lim _{t \rightarrow \infty} \Phi(t)=0
$$

\section{Energy estimates for solutions of the IBVP (8)-(13): A qualitative condition}

One common approach to studying stability of an equilibrium solution is to examine the linearized IBVP by considering the eigenvalue problem for the corresponding steady state BVP. Such an analysis gives rise to a polynomial of degree equal to the number of dependent variables in the system. Stability is then determined by the sign of the real parts of the roots of that polynomial. In a previous paper [12], we used this approach to examine a reduced system of three equations corresponding to (9)-(13). While this approach is effective, it has some disadvantages. First, it only determines that there exists initial data such that the resulting solution is unstable in time. Second, for large systems - say 4 by 4 or larger - the algebra can become highly cumbersome making it very difficult to track the impact of the coefficients on the signs of the roots of the polynomial.

In this article, we employ a different approach. Here, we will derive an appropriate energy functional and obtain those conditions underwhich this functional decays in time. 
We will present conditions on the coefficients of the system such that

$$
\int_{\Omega}\|\vec{U}(x, t)\|^{2} \mathrm{~d} x \rightarrow 0 \quad \text { as } t \rightarrow \infty
$$

where $\|\cdot\|$ is an appropriate norm to be specified later.

We begin our analysis by assuming that $\mu_{w}=0$. This is consistent with the immobile nature of debris within a lesion. Next, we assume that the impact of macrophages on the concentration of chemoattractant at equilibrium is negligible compared to diffusion $\left(\mu_{z}\right)$, production as an inflammatory effect $(K)$ and natural chemical degradation $(J)$. Mathematically, $a, \hat{a}<<1$. For simplicity, we will set $H=I=0$.

Now, we multiply Equation (8) by $E u$, (9) by $E v,(10)$ by $w,(11)$ by $E z$ and integrating by parts to obtain the system:

$$
\begin{gathered}
\partial_{t} \int E u^{2}=-\mu_{u} E \int|\nabla u|^{2}+\chi_{u} E \int \nabla z \nabla u-A E \int u^{2}-B E \int v u \\
\partial_{t} \int E v^{2}=-\mu_{v} E \int\left|\nabla^{2} v\right|^{2}+\chi_{v} E \int \nabla z \nabla v+A E \int u v+(B-C) E \int v^{2} \\
\partial_{t} \int w^{2}=-E \int u w+C \int v w-G \int w^{2} \\
\partial_{t} \int E z^{2}=-\mu_{z} E \int|\nabla z|^{2}+K E \int w z-J E \int z^{2} .
\end{gathered}
$$

We continue by imposing some constraints. The first is

CONSTRAint 1. All eigenvalues of the coefficent matrix

$$
\Lambda=\left[\begin{array}{cccc}
-A & -B & 0 & 0 \\
A & (B-C) & 0 & 0 \\
-E & C & -G & 0 \\
0 & 0 & K & -J
\end{array}\right]
$$

have negative real part. The matrix $\Lambda$ is the Transition matrix characterizing the species interaction. This first constraint ensures that integrals of the form $\int U_{i} \mathrm{~d} x \rightarrow 0$ as $t \rightarrow \infty$ for $U_{i}=u, v, w$ or $z$. The proof of this follows from Green's theorem and the homogeneous Neumann conditions imposed on each of the perturbation variables.

Note that Constraint 1 does not guarantee the stability of the system. That is, we can not conclude that $\int U_{i} \mathrm{~d} x \rightarrow 0$ implies that $U_{i} \rightarrow 0$ in time (or that we can even assume $U_{i}$ is bounded). See appendix A for a counter example.

Our construction will also make use of the additional constraint:

Constraint 2. Each of the parameters $A, B, C, E, G, K$ and $J$ are positive. Additionally,

$$
\Delta_{u}=\mu_{u}-\frac{\chi_{u}}{2}>0 \quad \text { and } \quad \Delta_{v}=\mu_{v}-\frac{\chi_{v}}{2}>0 .
$$

These last two terms $\Delta_{u}$ and $\Delta_{v}$ could be called net mobility parameters for the macrophage species. They are characteristic of the competing effects of diffusion which 
is stabilizing and chemotaxis which induces inflammation. The assumption that they are positive corresponds to control on inflammation consistant with a healthy configuration. We will impose the stronger condition that

$$
\mu_{u}>\chi_{u}
$$

In our derivation of the aforementioned norm, we will employ the inequalities

$$
\begin{aligned}
\text { (Cauchy) } a b & \leq \epsilon a^{2}+\frac{1}{4 \epsilon} b^{2}, \quad \text { and } \\
\text { (Poincaré) } \quad \int_{\Omega} u^{2} & \leq \frac{1}{|\Omega|}\left(\int_{\Omega} u\right)^{2}+C_{\mathrm{p}} \int_{\Omega}|\nabla u|^{2} .
\end{aligned}
$$

The parameter $C_{\mathrm{p}}$ is a constant that depends on the geometry of the domain. When an $L^{2}$ norm is considered, $C_{\mathrm{p}}$ is related to the inverse of the first positive eigenfrequency of a free membrane [1]. Because we have already imposed constraint, one which guarantees that integrals of the form $\int u$ vanish at infinity, we will make the simplifying assumption that the initial conditions of any perturbation of interest are orthogal to unity. This allows us to ignore integrals of the form $\int u$ from the beginning of the construction.

Next, we define the parameter $q_{0}$ via $G=q_{0} E$ (the purpose will become clear), combine the given constraints and use the above inequalities to obtain from Equations (14)-(17) the system of inequalities

$$
\begin{gathered}
\partial_{t} \int E u^{2} \leq \frac{p}{C_{\mathrm{p}}}\left(-\mu_{u}+\frac{\chi_{u}}{2}\right) E \int u^{2}-\gamma E \int u^{2}+\frac{B E}{2} \int v^{2}+\frac{\chi_{u}}{2} E \int|\nabla z|^{2}+(1-p) \\
\times\left(-\mu_{u}+\frac{\chi_{u}}{2}\right) E \int|\nabla u|^{2} \\
\partial_{t} \int E v^{2} \leq \frac{A E}{2} \int u^{2}+\left(\frac{1}{C_{\mathrm{p}}}\left(-\mu_{v}+\frac{\chi_{v}}{2}\right)+\frac{A}{2}+B-C\right) E \int v^{2}+\frac{\chi_{v}}{2} E \int|\nabla z|^{2} \\
\partial_{t} \int w^{2} \leq-E \int u w+\frac{C}{2} \int v^{2}+\left(\frac{C}{2}-q_{0} E\right) \int w^{2} \\
\partial_{t} \int E z^{2} \leq \frac{K E}{2} \int w^{2}+\left(\frac{K}{2}-J\right) E \int z^{2}-\mu_{z} E \int|\nabla z|^{2}
\end{gathered}
$$

Some like terms are separated for convenience. In this formulation, the parameter $p$ appearing in (18) satisfies $0<p<1$ and will be discussed in greater detail later. The parameter $\gamma$ in (18) is defined by $\gamma=A-(1 / 2) B$ and is assumed to be positive. Inequalities (18)-(21) hold for any choice of $p$ in $(0,1)$ and with a wide range of values of $\gamma$ as will be clarified later. We continue by obtaining a bound on the time derivative on a linear combination of integrals of the terms $u^{2}, v^{2}, w^{2}, z^{2}, u w,|\nabla u|^{2}$ and $|\nabla z|^{2}$.

After a number of calculations and applications of the aforementioned inequalities, we obtain a relation between the total spatial integral values of the quantities and their time derivatives (see appendix B for the details of the analysis). The resulting left-hand side 
(LHS) of the inequality is

$$
\mathrm{LHS}=\int \frac{\partial}{\partial t}\left[\left(E+\frac{A}{2}\right) u^{2}+E v^{2}+w^{2}+\left(E+\frac{J}{2}\right) z^{2}+u w+\frac{\mu_{u}}{2}|\nabla u|^{2}+\frac{\mu_{z}}{2}|\nabla z|^{2}\right] .
$$

On the right-hand side (RHS) of the resulting inequality, we will have coefficients of $\int u^{2}, \int v^{2}, \int w^{2}, \int z^{2}, \int u w, \int|\nabla u|^{2}$ and $\int|\nabla z|^{2}$ respectively,

$$
\begin{aligned}
& u^{2}:\left(1-r_{0}\right) \frac{p}{C_{\mathrm{p}}}\left(-\mu_{u}+\frac{\chi_{u}}{2}\right) E+\frac{A E+C}{2}-\left(E+\gamma E+r_{0} \frac{\mathrm{p}}{C_{\mathrm{p}}}\left(\mu_{u}-\frac{\chi_{u}}{2}\right) E\right) \\
& v^{2}: \frac{1}{C_{\mathrm{p}}}\left(-\mu_{v}+\frac{\chi_{v}}{2}\right) E+\frac{A E+3 B E+4 B^{2}-2 C E+2 C}{2} \\
& w^{2}: \frac{C+K E+1+2 K^{2}}{2}+2\left(\frac{K \chi_{u}}{\mu_{z}}\right)^{2}-\left(1-r_{1}\right) q_{0} E-\left(r_{1} q_{0} E\right) \\
& z^{2}: \frac{K E}{2}-J E+2\left(\frac{J \chi_{u}}{\mu_{z}}\right)^{2} \\
& u w:-\left(E+q_{0} E\right) \\
& |\nabla u|^{2}:(1-p)\left(-\mu_{u}+\frac{\chi_{u}}{2}\right) E \\
& |\nabla z|^{2}:-\mu_{z} E+\frac{\chi_{u}+\chi_{v}}{2} E
\end{aligned}
$$

Again we leave some like terms separated for convenience. The constants $r_{0}$ and $r_{1}$ satisfy $0<r_{0,1}<1$. The value $1 / 2$ that appears in (25) results from inequality (40) where it appears after applying the Cauchy inequality to the term $u_{t} w$. Although this is a fixed value, it appears as a parameter as a result of normalizing the coefficient of the time derivative in the original Equation (6).

Here, we return to the parameters introduced earlier, $\gamma$ and $p$. We wish to combine the final terms appearing in (23), (25) and (27), in such a way that

$$
\left(E+\gamma E+r_{0} \frac{p}{C_{\mathrm{p}}}\left(\mu_{u}-\frac{\chi_{u}}{2}\right) E\right) u^{2}+\left(E+q_{0} E\right) u w+\left(r_{1} q_{0} E\right) w^{2}=E(\alpha u+\beta w)^{2} .
$$

This requires that

$$
\alpha^{2}=\left(1+\gamma+r_{0} \frac{p}{C_{\mathrm{p}}}\left(\mu_{u}-\frac{\chi_{u}}{2}\right)\right), \quad \beta^{2}=r_{1} q_{0}
$$

and

$$
2 \alpha \beta=1+q_{0} .
$$


Thus, $\gamma$ must satisfy

$$
\gamma=\frac{\left(1+q_{0}\right)^{2}}{2 r_{1} q_{0}}-1-r_{0} \frac{p}{C_{\mathrm{p}}}\left(\mu_{u}-\frac{\chi_{u}}{2}\right)
$$

Given any values of $A, B$ and $q_{0}$, we can take $r_{0}$ and $r_{1}$ sufficiently small so that $\gamma>0$ as required.

A similar recombination of terms from the LHS gives

$$
\left(E+\frac{A}{2}\right) u^{2}+u w+w^{2}=\Theta u^{2}+\Psi w^{2}+\frac{1}{1+q_{0}}(\alpha u+\beta w)^{2}
$$

where the coefficients are given by

$$
\begin{aligned}
& \Theta=E+\frac{A}{2}-\frac{1}{1+q_{0}} \alpha^{2}=E+\frac{A}{2}-\frac{\left(1+q_{0}\right)}{4 r_{1} q_{0}} \\
& \text { and } \quad \Psi=1-\frac{1}{1+q_{0}} \beta^{2}=1-\frac{r_{1} q_{0}}{1+q_{0}} .
\end{aligned}
$$

We will require that these values are positive.

Next, we set $p=\left(\mu_{u}-\chi_{u}\right) /\left(2 \mu_{u}-\chi_{u}\right)$. Then $0<p<1$ as previously required and the coefficient of $|\nabla u|^{2}$ on the RHS of the inequality is

$$
(1-p)\left(-\mu_{u}+\frac{\chi_{u}}{2}\right)=-\frac{\mu_{u}}{2}
$$

which is proportional to the coefficient of $|\nabla u|^{2}$ on the left. Here, we are imposing the stronger condition on $\mu_{u}$ and $\chi_{u}$ as previously mentioned, namely $\mu_{u}>\chi_{u}$.

We are ready to write the completed inequality obtained by summing (18)-(21), (40), (42) and (44) (see the appendix for the latter three equations. Assuming that

$$
\frac{3}{4}-2\left(\frac{\chi_{u}}{\mu_{z}}\right)^{2} \geq 0
$$

all terms involving $\left(z_{t}\right)^{2}$ can be neglected. We have

$$
\begin{gathered}
\frac{\partial}{\partial t} \int\left[\Theta u^{2}+E v^{2}+\Psi w^{2}+\left(E+\frac{J}{2}\right) z^{2}+\frac{1}{1+q_{0}}(\alpha u+\beta w)^{2}+\frac{\mu_{u}}{2}|\nabla u|^{2}+\frac{\mu_{z}}{2}|\nabla z|^{2}\right] \\
\leq-C_{u} \int \Theta u^{2}-C_{v} \int E v^{2}-C_{w} \int \Psi w^{2}-C_{z} \int\left(E+\frac{J}{2}\right) z^{2} \\
\quad-C_{u w} \int \frac{1}{1+q_{0}}(\alpha u+\beta w)^{2}-C_{\nabla u} \int \frac{\mu_{u}}{2}|\nabla u|^{2}-C_{\nabla z} \int \frac{\mu_{z}}{2}|\nabla z|^{2} .
\end{gathered}
$$


The coefficients on the right are defined by

$$
\begin{aligned}
C_{u} & =\left(\left[\left(1-r_{0}\right) \frac{p}{C_{\mathrm{p}}}\left(\mu_{u}-\frac{\chi_{u}}{2}\right)-\frac{A}{2}\right] E-\frac{C}{2}\right) \frac{1}{\Theta} \\
C_{v} & =\frac{1}{C_{\mathrm{p}}}\left(\mu_{v}-\frac{\chi_{v}}{2}\right)+\frac{2 C-A-3 B}{2}-\frac{4 B^{2}+2 C}{2 E} \\
C_{w} & =\left(\left[\left(1-r_{1}\right) q_{0}-\frac{K}{2}\right] E-\frac{C+1+2 K^{2}}{2}-2\left(\frac{K \chi_{u}}{\mu_{z}}\right)^{2}\right) \frac{1}{\Psi} \\
C_{z} & =\left(\left[J-\frac{K}{2}\right] E-2\left(\frac{J \chi_{u}}{\mu_{z}}\right)^{2}\right) \frac{2}{2 E+J} \\
C_{u w} & =\left(1+q_{0}\right)^{2} E \\
C_{\nabla u} & =E \\
C_{\nabla z} & =\left(\mu_{z}-\frac{\chi_{u}+\chi_{v}}{2}\right) \frac{2 E}{\mu_{z}} .
\end{aligned}
$$

TheOrem 1 . The equilibrium solution $\left(m_{\mathrm{e}}, \hat{m}_{\mathrm{e}}, d_{\mathrm{e}}, c_{\mathrm{e}}\right)$ is asymptotically stable provided,

i. All eigenvalues of $\Lambda$ have negative real part,

ii. $\Delta_{u}>0$ and $\Delta_{v}>0$,

iii. $(3 / 4)-2\left(\frac{\chi_{u}}{\mu_{z}}\right)^{2} \geq 0, \Theta>0, \Psi>0$ and

iv. $M=\min \left\{C_{u}, C_{v}, C_{w}, C_{z}, C_{\nabla z}\right\}>0$

Proof. Define the functional

$$
\begin{gathered}
\mathcal{F}(\vec{U})=\int_{\Omega}\left[\Theta u^{2}+E v^{2}+\Psi w^{2}+\left(E+\frac{J}{2}\right) z^{2}+\frac{q_{0} E}{1+q_{0}}(\alpha u+\beta w)^{2}\right. \\
\left.+\frac{\mu_{u}}{2}|\nabla u|^{2}+\frac{\mu_{z}}{2}|\nabla z|^{2} \mathrm{~d} x\right]=\Phi(t)
\end{gathered}
$$

The conditions (i)-(iv) imply

$$
\frac{\mathrm{d}}{\mathrm{d} t} \Phi(t) \leq-M \Phi(t)
$$

so that $\Phi \rightarrow 0$ exponentially as $t \rightarrow \infty$.

To obtain a physical interpretion of this result we can consider the conditions $\mathrm{i}-\mathrm{iv}$ independently. First note that if the parameter $B \ll 1$ then the eigenvalues of $\Lambda$ can be approximated by

$$
\lambda_{1}=-A, \quad \lambda_{2}=-C, \quad \lambda_{3}=-G, \quad \lambda_{4}=-J .
$$


And so are all negative. The function $g_{0}$ depended on both subspecies of macrophages and when positive decreased the density of healthy macrophages and increased the number of apoptotic macrophages. The parameter $B=\partial_{\hat{m}} g_{0}$ (at equilibrium) is determined by the rate of apoptosis of macrophages due to apoptotic macrophage density. If this parameter is small, this would mean that at equilibrium the propensity for apoptosis induced by present apoptotic macrophages is weak. When $B$ is small, say

$$
B<A+C
$$

the eigenvalues will have negative real part. In terms of disease, this condition implies that the equilibrium density of apoptotic macrophages weakly influences the density of healthy macrophages.

Both conditions ii and iii hold when the diffusion coefficients are larger than the chemotactic sensitivity. So these can be interpreted as saying that diffusion rather than inflammation dominates.

To examine condition iv, we recall that $g_{1}$, which depends on the healthy macrophage density and the density of debris, describes the healthy immune response of the system. When positive, it corresponds to reduction of the debris density. The parameter $E=\partial_{m} g_{1}$ (at equilibrium), is the rate at which debris is removed as it depends on viable macrophage density. Upon examination of each of the parameters that appear in condition iv, we see that positivity of each depends on $E$ being large. Note that the positive part of each parameter in the set appearing in condition iv depends on $E$ (except for $C_{v}$ in which the negative part depends inversely on $E$ ). The minimum $M>0$ corresponds to large $E$ which corresponds to a strong healthy immune response. This would indicate that the level of immune response is appropriate as opposed to the runaway inflammatory characteristic of disease.

As $q_{0}>0$ and $r_{1}<1$ the parameter $\Psi$ is positive by definition. The condition that $\Theta>0$ gives a weak requirement between $q_{0}$ and $E$. Since $r_{0}$ can be made as small (but positive) as desired, the condition $\gamma>0$ requires that

$$
r_{1}<\frac{\left(1+q_{0}\right)^{2}}{2 q_{0}}
$$

Assuming that $r_{1} \approx\left(1+q_{0}\right)^{2} /\left(2 q_{0}\right)$, the condition on $\Theta$ is approximated by the condition on $E$

$$
E+\frac{A}{2}>\frac{1}{2\left(1+q_{0}\right)}
$$

We can eliminate both of these conditions and obtain a weaker stability result. If we assume that $E>1$ and set $E=\bar{E}+1$, we can replace (30) with

$$
\left(E+\frac{A}{2}\right) u^{2}+u w+w^{2}=\left(\bar{E}+\frac{A}{2}\right) u^{2}+w^{2}+\frac{1}{2}\left(\sqrt{2} u+\frac{1}{\sqrt{2}} w\right)^{2}
$$


The inequality (31) will be replaced by the inequality

$$
\begin{aligned}
\frac{\partial}{\partial t} \int\left[\left(\bar{E}+\frac{A}{2}\right) u^{2}+E v^{2}+w^{2}+\left(E+\frac{J}{2}\right) z^{2}\right. \\
\left.+\frac{1}{2}\left(\sqrt{2} u+\frac{1}{\sqrt{2}} w\right)^{2}+\frac{\mu_{u}}{2}|\nabla u|^{2}+\frac{\mu_{z}}{2}|\nabla z|^{2}\right] \\
\quad \leq-C_{u} \int\left(\bar{E}+\frac{A}{2}\right) u^{2}-C_{v} \int E v^{2}-C_{w} \int w^{2}-C_{z} \int\left(E+\frac{J}{2}\right) z^{2} \\
\quad-C_{u w} \int \frac{1}{1+q_{0}}(\alpha u+\beta w)^{2}-C_{\nabla u} \int \frac{\mu_{u}}{2}|\nabla u|^{2}-C_{\nabla z} \int \frac{\mu_{z}}{2}|\nabla z|^{2},
\end{aligned}
$$

with the coefficients on the right the same as before with the exceptions

$$
\begin{aligned}
C_{u} & =\left(\left[\left(1-r_{0}\right) \frac{p}{C_{\mathrm{p}}}\left(\mu_{u}-\frac{\chi_{u}}{2}\right)-\frac{A}{2}\right] E-\frac{C}{2}\right) \frac{2}{2 \bar{E}+A} \\
C_{w} & =\left(\left[\left(1-r_{1}\right) q_{0}-\frac{K}{2}\right] E-\frac{C+1+2 K^{2}}{2}-2\left(\frac{K \chi_{u}}{\mu_{z}}\right)^{2}\right) .
\end{aligned}
$$

Under these conditions, the norms on the left and right sides of the inequalities differ, but only in the square of the linear combination of $u$ and $w$. We can state the following stability criteria (all variables except for $C_{u}$ and $C_{w}$ are as previously defined):

Theorem 2. The equilibrium solution $\left(m_{\mathrm{e}}, \hat{m}_{\mathrm{e}}, d_{\mathrm{e}}, c_{\mathrm{e}}\right)$ is stable provided,

i. All eigenvalues of $\Lambda$ have negative real part,

ii. $\Delta_{u}>0$ and $\Delta_{v}>0$,

iii. $(3 / 4)-2\left(\frac{\chi_{u}}{\mu_{z}}\right)^{2} \geq 0, E>1$ and

iv. $M=\min \left\{C_{u}, C_{v}, C_{w}, C_{z}, C_{\nabla z}\right\}>0$

The image of the functional $\Phi(t)$ obtained here would satisfy

$$
\frac{\mathrm{d}}{\mathrm{d} t} \Phi(t) \leq 0
$$

It is not difficult to show that each integral

$$
\int u^{2}, \quad \int v^{2}, \quad \int w^{2}, \quad \int z^{2},
$$

will vanish as $t \rightarrow \infty$. However, we cannot in this case guarantee exponential decay.

\section{Analysis of a mathematically interesting special parameter case}

Under the special case that the parameters $E$ and $G$ are equal, we can derive a sufficient condition for stability that is (although not necessary) strong in the sense that the inequality is tight. We begin the construction similar to the previous one except that the initial inequalities are obtained by mulitplying Equation (8) by $u,(9)$ by $v,(10)$ by $w,(11)$ 
by $z$ and integrating by parts. The inequality that we obtain after computation is

$$
\begin{gathered}
\frac{\partial}{\partial t} \int\left[\frac{A}{2} u^{2}+v^{2}+\frac{3}{4} w^{2}+\frac{1}{2}\left(\sqrt{2} u+\frac{1}{\sqrt{2}} w\right)^{2}+\left(1+\frac{J}{2}\right) z^{2}+\frac{\mu_{u}}{2}|\nabla u|^{2}+\frac{\mu_{z}}{2}|\nabla z|^{2}\right] \\
\leq-C_{u} \int \frac{A}{2} u^{2}-C_{v} \int v^{2}-C_{w} \int \frac{3}{4} w^{2}-C_{z} \int\left(1+\frac{J}{2}\right) z^{2} \\
\quad-C_{u w} \int \frac{1}{2}\left(\sqrt{2} u+\frac{1}{\sqrt{2}} w\right)^{2}-C_{\nabla u} \int|\nabla u|^{2}-C_{\nabla z} \int \frac{\mu_{z}}{2}|\nabla z|^{2}
\end{gathered}
$$

In this construction, the coefficients on the right are given by

$$
\begin{aligned}
C_{u} & =\left(\frac{p}{C_{\mathrm{p}}}\left(\mu_{u}-\frac{\chi_{u}}{2}\right)-\frac{A+C+D}{2}\right) \frac{2}{A} \\
C_{v} & =\frac{1}{C_{\mathrm{p}}}\left(\mu_{v}-\frac{\chi_{v}}{2}\right)-\frac{A+3 B+4 B^{2}+D}{2} \\
C_{w} & =\frac{E}{3}-\left(\frac{C+4 D+K+1+2 K^{2}}{2}+2\left(\frac{K \chi_{u}}{\mu_{z}}\right)^{2}\right) \frac{4}{3} \\
C_{z} & =\left(J-\frac{K}{2}-2\left(\frac{J \chi_{u}}{\mu_{z}}\right)^{2}\right) \frac{2}{2+J} \\
C_{u w} & =E \\
C_{\nabla u} & =\frac{\mu_{u}}{2} \\
C_{\nabla z} & =\left(\mu_{z}-\frac{\chi_{u}+\chi_{v}}{2}\right) \frac{2}{\mu_{z}} .
\end{aligned}
$$

This is the result that is the analogue of the inequality (31). We can state the following theorem, the proof is the same as for Theorem 1:

TheOrem 3. The equilibrium solution $\left(m_{\mathrm{e}}, \hat{m}_{\mathrm{e}}, d_{\mathrm{e}}, c_{\mathrm{e}}\right)$ is asymptotically stable provided,
i. All eigenvalues of $\Lambda$ have negative real part,
ii. $\Delta_{u}>0$ and $\Delta_{v}>0$,
iii. $(3 / 4)-2\left(\frac{\chi_{u}}{\mu_{z}}\right)^{2} \geq 0$ and
iv. $M=\min \left\{C_{u}, C_{v}, C_{w}, C_{z}, C_{\nabla z}\right\}>0$. 
The matrix $\Lambda$ is

$$
\Lambda=\left[\begin{array}{cccc}
-A & -B & 0 & 0 \\
A & (B-C) & 0 & 0 \\
-E & C & -E & 0 \\
0 & 0 & K & -J
\end{array}\right] .
$$

This is the same as the previous definition with $G=E$.

For this case, we claim that conditions $\mathrm{i}-\mathrm{iv}$ are strong stability criteria. We begin by noting that if one of the eigenvalues of the matrix $\Lambda$ has positive real part, then at least one of the integrals

$$
\int_{\Omega} u \mathrm{~d} x, \quad \int_{\Omega} v \mathrm{~d} x, \quad \int_{\Omega} w \mathrm{~d} x, \quad \text { or } \quad \int_{\Omega} z \mathrm{~d} x
$$

will tend to infinity. This can be seen by noting that if we integrate the system (8)-(11) over $\Omega$ and apply the Neumann boundary condition, each of the integrals will be exponential in time with exponents depending on the eigenvalues of $\Lambda$.

To show the importance of conditions ii-iv, let us suppose that the rate of removal of debris due to healthy macrophages (healthy immune response) $E$ is large compared with other reaction terms, say

$$
E>4\left(C+4 D+K+1+2 K^{2}\right),
$$

and that the rate of degradation of the chemoattractant is comparable with the rate of its production

$$
J>\frac{K}{2} .
$$

This helps to ensure that $C_{w}>0$ and that $C_{z}>0$ provided $\mu_{z}$ which describes diffusive effects is large. Now, the three conditions ii-iv imply domination of the stabilizing diffusive effects over the chemotactic forces. To show that these bounds are tight let us set

$$
u=u_{0} \mathrm{e}^{\sigma t} \phi_{\lambda}(x), \quad v=v_{0} \mathrm{e}^{\sigma t} \phi_{\lambda}(x), \quad w=w_{0} \mathrm{e}^{\sigma t} \phi_{\lambda}(x) \quad \text { and } \quad z=z_{0} \mathrm{e}^{\sigma t} \phi_{\lambda}(x),
$$

where $\phi_{\lambda}(x)$ is the first nonconstant solution of the Neumann problem (38) corresponding to the first positive eigenvalue $\lambda$. Let $B=0$ and set $\mu_{u}=\mu_{v}=\mu$ (i.e. we assume that random motion of macrophages is the same regardless of the subspecies). We will show that under these assumptions and some conditions on the remaining coefficients $A, C$ and $E$ that for any $\mu, \mu_{z}, \chi_{u}$ and $\lambda$ (which corresponds to the size of the domain), there exists $\chi_{v}$ sufficiently large such that the solution of the linearized IBVP will not decay with time. 
Substitution $u, v, w, z$ into the system (8)-(11) results in the algebraic system of equations

$$
\left(\begin{array}{cccc}
(\sigma+\mu \lambda+A) & 0 & 0 & -\chi_{u} \lambda \\
-A & (\sigma+\mu \lambda+C) & 0 & -\chi_{v} \lambda \\
E & -C & (\sigma+E) & 0 \\
0 & 0 & -K & \left(\sigma+\mu_{z} \lambda+J\right)
\end{array}\right)\left(\begin{array}{c}
u_{0} \\
v_{0} \\
w_{0} \\
z_{0}
\end{array}\right)=0 .
$$

A nontrivial solution $\left(u_{0}, v_{0}, w_{0}, z_{0}\right)^{T}$ exists if and only if the determinant of the coefficient matrix is zero. Letting

$$
\Delta=\left|\begin{array}{cccc}
(\sigma+\mu \lambda+A) & 0 & 0 & -\chi_{u} \lambda \\
-A & (\sigma+\mu \lambda+C) & 0 & -\chi_{v} \lambda \\
E & -C & (\sigma+E) & 0 \\
0 & 0 & -K & \left(\sigma+\mu_{z} \lambda+J\right)
\end{array}\right|,
$$

we have

$$
\begin{aligned}
\Delta & =(\sigma+\mu \lambda+A)\left[(\sigma+\mu \lambda+C)\left(\sigma+\mu_{z} \lambda+J\right)(\sigma+E)-\lambda \chi_{\nu} C K\right] \\
& -\lambda \chi_{u} K[A C-(\sigma+\mu \lambda+C) E] .
\end{aligned}
$$

Next, we set $C=2 A$ and $E=2 A$. Then the last term in (37) is

$$
A C-(\sigma+\mu \lambda+C) E=2 A^{2}-2(\sigma+\mu \lambda+2 A) A=-2 A(\sigma+\mu \lambda+A) .
$$

Replacing the last term in (37) we have

$$
\begin{aligned}
\Delta & =(\sigma+\mu \lambda+A)\left[(\sigma+\mu \lambda+C)\left(\sigma+\mu_{z} \lambda+J\right)(\sigma+E)-\lambda \chi_{\nu} C K+2 A \lambda \chi_{u} K\right] \\
& =(\sigma+\mu \lambda+A) P_{3}(\sigma),
\end{aligned}
$$

where the polynomial $P_{3}$ has the form

$$
P_{3}(\sigma)=\sigma^{3}+a_{2} \sigma^{2}+a_{1} \sigma+(\mu \lambda+C) E\left(\mu_{z} \lambda+J\right)+2 A \lambda \chi_{u} K-\lambda \chi_{\nu} C K .
$$

Now each of the coefficients $a_{1}$ and $a_{2}$ are products of the terms $\mu, \mu_{z}, \lambda, C, E, J$ and $K$, all of which are positive. Hence, $a_{1}>0$ and $a_{2}>0$.

We know that for the equilibrium solution to the IBVP to be stable, all roots of $P_{3}$ must have negative real part. As stated earlier, we will show that there exists $\chi_{v}$ sufficiently large such that for any positive $\mu, \mu_{z}, \chi_{u}, \lambda, A, E, J$ and $K$ at least one root has positive real part.

To this end we let the constant term in the polynomial be denoted by $a_{0}$. By the RouthHurwitz criteria [6], $P_{3}$ is guaranteed to have at least one root with positive real part if $a_{0}<0$. It is sufficient to take

$$
\chi_{v}>\frac{(\mu \lambda+C) E\left(\mu_{z} \lambda+J\right)+2 A \lambda \chi_{u} K}{\lambda C K} .
$$

Hence, we conclude stability depends strongly on the criteria i-iv. 


\section{Conclusion}

In our recent work [12] we showed that consideration of the onset of an atherosclerotic lesion as the result of a mathematical instability in an otherwise healthy steady distribution of cells gives rise to physically meaningful stability criterion. Here, we have extended our analysis to better allow for the complex process of both inflammation and foam cell production by accounting for simultaneous existence immune cells to be in different stages of health and disease. While this increases the number of equations under consideration, and thus the difficulty involved in the mathematical analysis, it also increases our ability to arrive at meaningful bio-physical conclusions.

Through an energy estimate approach, we arrived at sufficient and strong conditions for stability. Collectively, these conditions imply that for the system to be resistant to lesion formation any course of action that increases the diffusivity of the tissue and allows for moderate and controlled inflammation should be taken.

The flexibility of the approach taken here is of significance aside from the particular stability criteria obtained for the system of interest. We offer that this method could be modified to focus on any parameter of note. The approach is also readily adapted to larger systems of equations because it does not require analysis of a high order polynomial.

\section{References}

[1] G. Acosta and R.G. Durán, An optimal Poincaré inequality in $L^{1}$ for convex domains, Proc. Am. Math. Soc. 132(1) (2003), pp. 195-202.

[2] C.G. Caro, J.M. Fitz-Gerald, and M.F. Schroter, Atheroma and arterial wall shear: Observation, correlation and proposal of a shear dependent mass transfer mechanism for atherogenesis, Proc. R. Soc. Lond. B 177 (1971), pp. 109-133.

[3] A. Chait et al., Lipoprotein-associated inflammatory proteins: Markers or mediators of cardiovascular disease?, J. Lipid Res. 46 (2005), pp. 389-403.

[4] C.A. Cobbold, J.A. Sherratt, and S.J.R. Maxwell, Lipoprotein oxidation and its significance for atherosclerosis: A mathematical approach, Bull. Math. Biol. 64 (2002), pp. 65-95.

[5] D. Cui et al., Pivital advance: Macrophages become resistant to cholesterol-induced death after phagocytosis of apoptotic cells, J. Leukoc. Biol. 82 (2007), pp. 1040-1050.

[6] F.R. Gantmacher (Translated from Russian by K.A. Hirsch) In The Theory of Matrices, vol. I, Chelsea, New York, 1959, p. $10+374$

[7] J.L. Goldstein et al., Binding site on macrophages that mediates uptake and degradation of acetylated low density lipoproteins, producing massive cholesterol deposition, Proc. Natl. Acad. Sci. USA 76 (1977), pp. 333-337.

[8] D.P. Hajjar and M.E. Haberland, Lipoprotein trafficking in vascular cells; molecular trojan horses and cellular saboteurs, J. Biol. Chem. 272 (1997), pp. 22975-22978.

[9] G.A. Holzapfel, T.C. Gasser, and R.W. Ogden, A new constitutive framework for arterial wall mechanics and a comparative study of material models, J. Elasticity 61 (2000), pp. 1-48.

[10] D. Horstmann, From 1970 until present: The Keller-Segel model in chemotaxis and its consequences, Jahresber. Deutsch. Math. Verein. 105(3) (2003), pp. 103-165.

[11] A.I. Ibragimov et al., A mathematical model of atherogenesis as an inflammatory response, Math. Med. Biol. 22 (2005), pp. 305-333.

[12] A.I. Ibragimov et al., A dynamic model of atherogenesis as an inflammatory response, Dyn. Contin. Discrete Impuls. Syst. A Supplement, Advances in Dynamical Systems 14(52) (2007), pp. 185-189.

[13] E.F. Keller and L.A. Segel, Model for chemotaxis, J. Theor. Biol. 30 (1971), pp. 235-248.

[14] R. Kinscherf et al., Characterization of apoptotic macrophages in atheromatous tissue of humans and heritable hyperlipidemic rabbits, Atherosclerosis 144(1) (1999), pp. 33-39.

[15] N. Mantzaris, S. Webb, and H.G. Othmer, Mathematical modeling of tumor-induced angiogenesis, J. Math. Biol. 49(2) (2004), pp. 111-187.

[16] E.A. Podrez et al., Identification of a novel family of oxidized phospholipids that serve as ligands for the macrophage scavenger receptor CD36, J. Biol. Chem. 277(41) (2002), pp. 38503-38516.

[17] R. Ross, Cell biology of atherosclerosis, Annu. Rev. Physiol. 57 (1995), pp. $791-804$.

[18] R. Ross, Atherosclerosis - an inflammatory disease, N. Engl. J. Med. 340(2) (1999), pp. $115-126$.

[19] D. Steinberg, Low density lipoprotein oxidation and its pathobiological significance, J. Biol. Chem. 272 (1997), pp. 20963-20966. 
[20] R. Stocker and J. Keaney, Role of oxidative modifications in atherosclerosis, Physiol. Rev. 84 (2004), pp. $1381-1478$.

[21] I. Tabas, Apoptosis and plaque destabilization in atherosclerosis: The role of macrophage apoptosis induced by cholesterol, Cell Death Differ. 11 (2004), pp. S12-S16.

\section{Appendix A: Contraint 1 does not guarantee stability of the system (8)-(13)}

Suppose that we assume that $A, C, E, G, K$ and $J$ are positive and that $B=0$. Then it is obvious that all eigenvalues of $\Lambda$ are negative; Constraint 1 is satisfied. Next, let $\phi_{\lambda}(x)$ be an eigenfunction of the Neumann problem

$$
\nabla^{2} \phi_{\lambda}(x)=-\lambda \phi_{\lambda}(x) \quad x \in \Omega \quad \frac{\partial \phi_{\lambda}}{\partial \vec{n}} \quad \text { on } \quad \partial \Omega
$$

Put $u=v=w=z=\mathrm{e}^{\sigma t} \phi_{\lambda}(x)$ and substitute them into system (8)-(13). The PDE is reduced to the algebraic system:

$$
\begin{aligned}
& \sigma=\left(-\mu_{u}+\chi_{u}\right) \lambda-A \\
& \sigma=\left(-\mu_{v}+\chi_{v}\right) \lambda+A-C \\
& \sigma=-E-G+C \\
& \sigma=-\mu_{z}+K-J .
\end{aligned}
$$

It is not difficult to show that there exists a set of coefficients for this system such that $\sigma>0$. As an example, let $\lambda$ be any positive eigenvalue and set

$$
A=1, \quad C=2, \quad E=G=\frac{1}{2}, \quad J=2, \quad K=1, \quad \chi_{u}=\chi_{v}=\chi=\frac{4}{\lambda}, \quad \mu_{u}=\mu_{v}=\mu_{z}=\frac{\chi}{2}
$$

Then $\sigma=1$.

\section{Appendix B: Derivation of (31)}

We began by multiplying Equation (8) by $E u$, (9) by $E v$, (10) by $w,(11)$ by $E z$ and integrating by parts to obtain the system (14)-(17) in Section 5:

$$
\begin{gathered}
\partial_{t} \int E u^{2}=-\mu_{u} E \int|\nabla u|^{2}+\chi_{u} E \int \nabla z \nabla u-A E \int u^{2}-B E \int v u \\
\partial_{t} \int E v^{2}=-\mu_{v} E \int\left|\nabla^{2} v\right|^{2}+\chi_{v} E \int \nabla z \nabla v+A E \int u v+(B-C) E \int v^{2} \\
\partial_{t} \int w^{2}=-E \int u w+C \int v w-G \int w^{2} \\
\partial_{t} \int E z^{2}=-\mu_{z} E \int|\nabla z|^{2}+K E \int w z-J E \int z^{2} .
\end{gathered}
$$

Our computations will take into account the constraints listed - the eigenvalues of the transition matrix $\Lambda$ have negative real part and that the net mobility parameters are positive. We defined the 
parameter $q_{0}$ as the ratio of $G$ to $E$ and obtained our initial inequalties (18)-(21)

$$
\begin{aligned}
& \partial_{t} \int E u^{2} \leq \frac{p}{C_{\mathrm{p}}}\left(-\mu_{u}+\frac{\chi_{u}}{2}\right) E \int u^{2}-\gamma E \int u^{2}+\frac{B E}{2} \int v^{2} \\
&+\frac{\chi_{u}}{2} E \int|\nabla z|^{2}+(1-p)\left(-\mu_{u}+\frac{\chi_{u}}{2}\right) E \int|\nabla u|^{2} \\
& \partial_{t} \int E v^{2} \leq \frac{A E}{2} \int u^{2}+\left(\frac{1}{C_{\mathrm{p}}}\left(-\mu_{v}+\frac{\chi_{v}}{2}\right)+\frac{A}{2}+B-C\right) E \int v^{2}+\frac{\chi_{v}}{2} E \int|\nabla z|^{2} \\
& \partial_{t} \int w^{2} \leq-E \int u w+\frac{C}{2} \int v^{2}+\left(\frac{C}{2}-q_{0} E\right) \int w^{2} \\
& \partial_{t} E \int z^{2} \leq \frac{K E}{2} \int w^{2}+\left(\frac{K}{2}-J\right) E \int z^{2}-\mu_{z} E \int|\nabla z|^{2}
\end{aligned}
$$

We require bounds on a number of terms to arrive at (31). To that end, we multiply Equation (10) by $u$, add $u_{t} w$ to both sides of the result, and integrate to obtain

$$
\partial_{t} \int(u w)=-E \int u^{2}+C \int u v-q_{0} E \int(u w)+\int u_{t} w
$$

Applying the Cauchy inequality to this last equation yields

$$
\partial_{t} \int(u w) \leq-E \int u^{2}+\frac{C}{2} \int u^{2}+\frac{C}{2} \int v^{2}-q_{0} E \int(u w)+\frac{1}{2} \int w^{2}+\frac{1}{2} \int\left(u_{t}\right)^{2} .
$$

We desire a bound on the last term in (40). To that end, we multiply both sides of (8) by $u_{t}$ and integrate by parts.

$$
\int\left(u_{t}\right)^{2}=-\frac{\mu_{u}}{2} \frac{\partial}{\partial t} \int|\nabla u|^{2}-\chi_{u} \int u_{t} \nabla^{2} z-\frac{A}{2} \int\left(u^{2}\right)_{t}-B \int u_{t} v
$$

Next, we replace $\nabla^{2} z$ appearing on the RHS with $\left(1 / \mu_{z}\right)\left(z_{t}-K w+J z\right)$ which is obtained from (11), and apply the Cauchy inequality. We note here that

$$
\begin{aligned}
-B u_{t} v-\frac{\chi_{u}}{\mu_{z}} u_{t}\left(z_{t}-K w+J z\right) & \leq \frac{1}{8}\left(u_{t}\right)^{2}+2 B^{2} v^{2}+\frac{1}{8}\left(u_{t}\right)^{2}+2\left(\frac{\chi_{u}}{\mu_{z}}\right)^{2}\left(z_{t}\right)^{2}+\frac{1}{8}\left(u_{t}\right)^{2} \\
& +2\left(\frac{K \chi_{u}}{\mu_{z}}\right)^{2} w^{2}+\frac{1}{8}\left(u_{t}\right)^{2}+2\left(\frac{J \chi_{u}}{\mu_{z}}\right)^{2} z^{2} .
\end{aligned}
$$

This is obtained by repeated use of the Cauchy inequality taking in turn $\epsilon=1 / 8 B, \epsilon=\mu_{z} / 8 \chi_{u}$, $\epsilon=\mu_{z} / 8 K \chi_{u}$ and $\epsilon=\mu_{z} / 8 J \chi_{u}$. Thus, Equation (41) gives us the inequality

$$
\begin{aligned}
\frac{1}{2} \int\left(u_{t}\right)^{2} \leq- & \frac{\mu_{u}}{2} \frac{\partial}{\partial t} \int|\nabla u|^{2}-\frac{A}{2} \int\left(u^{2}\right)_{t}+2 B^{2} \int v^{2}+2\left(\frac{\chi_{u}}{\mu_{z}}\right)^{2} \int\left(z_{t}\right)^{2} \\
+ & 2\left(\frac{K \chi_{u}}{\mu_{z}}\right)^{2} \int w^{2}+2\left(\frac{J \chi_{u}}{\mu_{z}}\right)^{2} \int z^{2}
\end{aligned}
$$


It remains to obtain a bound on $z_{t}$. To this end, we multipy Equation (11) by $z_{t}$, integrate by parts and apply the Cauchy inequality to get

$$
\frac{3}{4} \int\left(z_{t}\right)^{2} \leq-\frac{\mu_{z}}{2} \frac{\partial}{\partial t} \int|\nabla z|^{2}+K^{2} \int w^{2}-\frac{J}{2} \int\left(z^{2}\right)_{t}
$$

Let us sum the inequalities (18)-(21), (40), (42) and (43). In so doing, we place all terms involving a time derivative except for those involving $\left(z_{t}\right)^{2}$ to the left side of the inequality. The resulting LHS as it appears in Section 5 is

$$
\text { LHS }=\int \frac{\partial}{\partial t}\left[\left(E+\frac{A}{2}\right) u^{2}+E v^{2}+w^{2}+\left(E+\frac{J}{2}\right) z^{2}+u w+\frac{\mu_{u}}{2}|\nabla u|^{2}+\frac{\mu_{z}}{2}|\nabla z|^{2}\right] .
$$




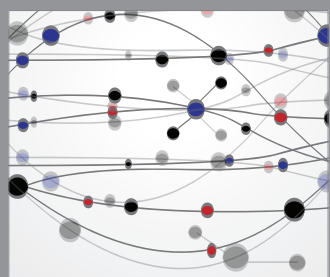

The Scientific World Journal
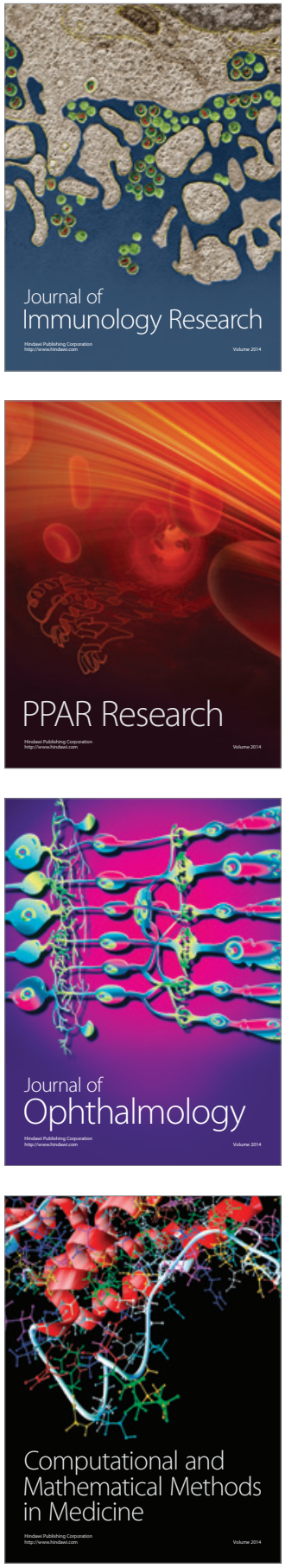

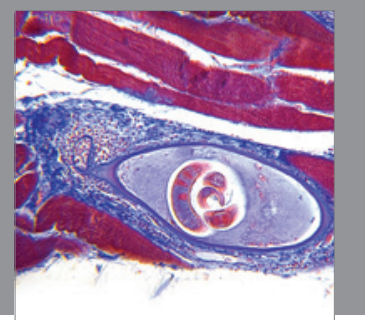

Gastroenterology

Research and Practice
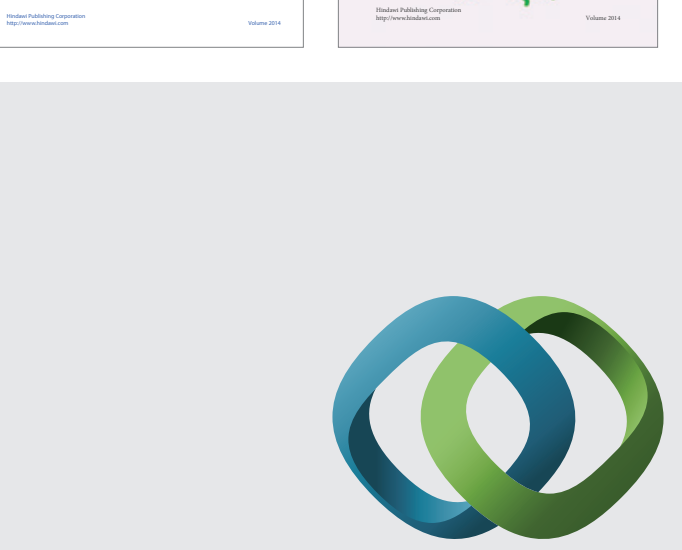

\section{Hindawi}

Submit your manuscripts at

http://www.hindawi.com
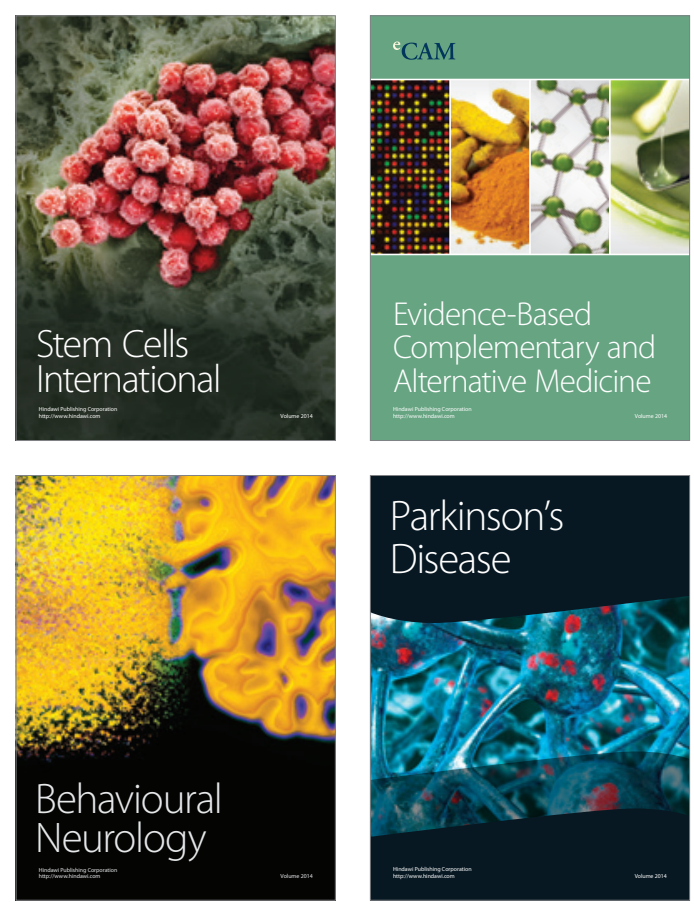

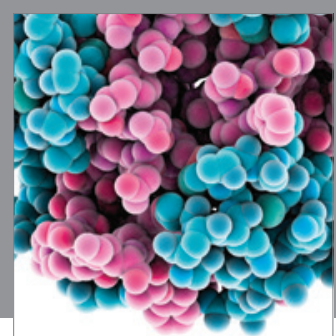

Journal of
Diabetes Research

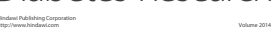

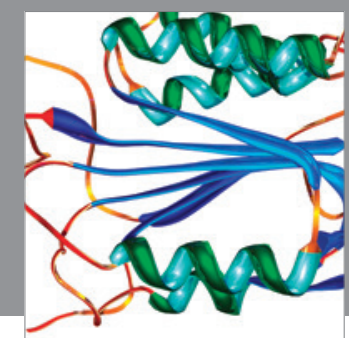

Disease Markers
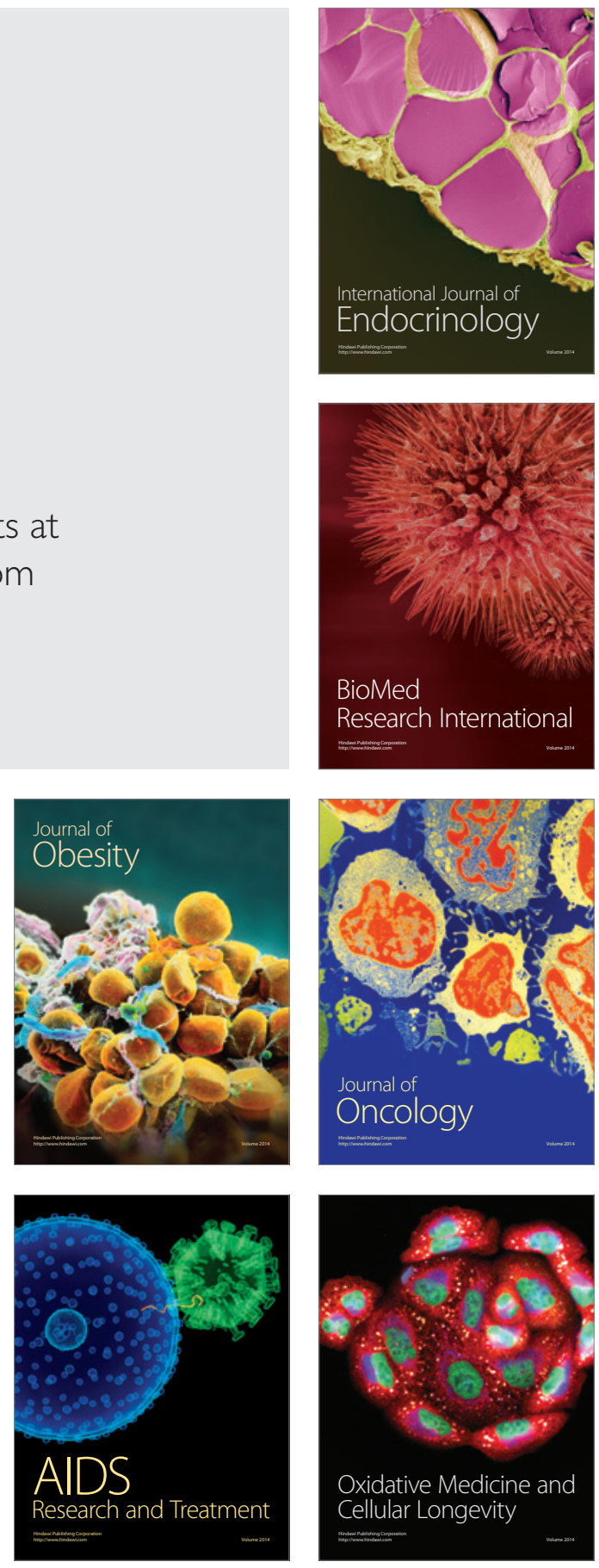